\title{
Evolutions des places et rôles du patient
}

\section{Jean Martin}

Dr med., membre de la rédaction

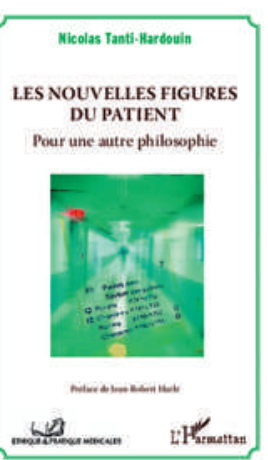

Nicolas Tanti-

Hardouin

Les nouvelles

figures du patient

Pour une autre philosophie Paris: L’Harmattan; 2014

177 pages. $24.50 \mathrm{CHF}$. ISBN 978-2-343-04760-7
Nicolas Tanti-Hardouin est un économiste enseignant les sciences sociales de la santé à la Faculté de médecine de Marseille. Dans cet ouvrage, il présente et analyse les mutations de l'image du patient, telle que la voient les praticiens, les observateurs du système de santé, les malades et leurs associations, notamment. Il décrit et commente différents profils ou portraits: l'homo medicus, patient rationnel de comportement idéal (le "bon patient»); le malade "sachant», porteur d'un savoir profane mais mieux informé qu'auparavant de la santé, de ses troubles et de la réalité du système de santé et apte à exprimer des hypothèses (rôle évidemment des possibilités ouvertes par internet); le patient consommateur de soins, qui devient client, faisant jouer la concurrence; le sujet-patient, produit de l'«avènement médical du sujet», voulant être reconnu comme singulier, avec en pleine lumière son autonomie, «véritables norme des normes éthiques pour ceux qui la revendiquent», allant vers le "self-care»; dans la foulée vient le patient capable avec l'acquisition de l'«empowerment», notamment dans la maladie chronique telle que le diabète; enfin le patient vulnérable dont l'autonomie est brisée et qu'il faudrait reconstituer. On voit par cet exposé que l'autonomie, principe et problématique qui occupe énormément aujourd'hui professionnels de santé comme philosophes et éthiciens, peut prendre des formes diverses, selon qu'on parle d'un sujet sain ou presque ou d'un malade sérieusement atteint («de multiples témoignages attestent que la maladie grave transforme l'identité du patient»).

Tanti-Hardouin présente ces thèmes au travers d'un parcours impressionnant de la littérature pertinente des dernières décennies. La bibliographie sur laquelle il se base est extensive, pour la plupart d'auteurs français mais sans ignorer les apports anglo-saxons. A noter l'importance quantitative des citations d'autres auteurs. En soi une bonne chose; toutefois, elles représentent (approximation) un tiers du texte et sont en italiques, et je n'ai pas trouvé agréable le constant passage de paragraphes en caractères droits (ce qu'a rédigé l'auteur) aux citations en italiques. Ceci fait un peu de l'ouvrage, en résumé, une anthologie de la littérature récente en sociologie de la santé, commentée par l'expert - et collègue - qu'est l'auteur. Cette structure, ce format, étant admis, c'est complet et intéressant.

Dans la dernière partie, Tanti-Hardouin évoque la problématique de l'influence des contraintes financières sur les soins (la médecine n'a pas de prix mais elle a un coût / un budget). Il demande si la santé publique avec ses professionnels et ses programmes, «imprégnée d'économicité», n’a pas à cet égard des conséquences regrettables. Là, le médecin de santé publique qui rédige cette recension est interpelé: dire qu'on devrait ne pas laisser une dimension (la mise à disposition des ressources nécessaires à la prise en charge de la maladie et de l'accident) "contaminer» la dispensation quotidienne, relationnelle et empathique, des soins, c'est un très bon sujet de débat voire de harangue politique. Mais comment imaginer qu'une telle séparation/étanchéité soit faisable dans des régimes socio-politiques comme ceux de l'Europe occidentale; où, tout en voulant garder des caractéristiques d'exercice libéral, le système est très largement financé par des ressources publiques? A mon sens, pour l'essentiel nous sommes condamnés à "faire au mieux", en préservant la liberté thérapeutique dans le cadre des moyens décidés par le politique, représentant - pour le moins idéalement de la communauté.

L'ouvrage bénéficie d'une intéressante préface de Jean-Robert Harlé, chef d'un Département de médecine interne à Marseille. Extrait: "Ce début de $\mathrm{XXI}^{\mathrm{e}}$ siècle est celui de la transformation de la relation entre malade et soignants [...]: des lois nouvelles [en France, notamment celle dite loi Kouchner, du 4 mars 2002, sur le droit des malades] et les nouvelles organisations sanitaires [...] ont profondément bousculé le caricatural paternalisme médical, ce qui laisse les soignants un peu perplexes (et un peu nostalgiques?). La dialectique du pouvoir et son partage restent cependant bien présents.» Et plus loin: "La distance séparant le 'bon patient' de la réalité ressentie sur le terrain donne la mesure du travail à accomplir, tant en termes d'éducation thérapeutique que [de prise en compte] de la vulnérabilité des patients.» 